\title{
Common Side Effects of COVID-19 Vaccines Used in Iran: A Review
}

\author{
Sepehr Siahvoshi (iD) ${ }^{1}$, Emad Movahed (iD) ${ }^{1,}$ and Kimia Ghods (iD) ${ }^{1}$ \\ ${ }^{1}$ Dental Materials Research Center, Dental School, Islamic Azad University of Medical Sciences, Tehran, Iran \\ "Corresponding author: Dental Materials Research Center, Dental School, Islamic Azad University of Medical Sciences, Tehran, Iran. Email: emovahed@outlook.com
}

Received 2021 October 15; Revised 2021 December 20; Accepted 2021 December 26.

\begin{abstract}
Context: Iran was one of the first countries in the world to encounter acute respiratory syndrome coronavirus 2 in February 2020. Numerous efforts have been made worldwide to develop safe and effective vaccines to prevent coronavirus disease 2019 (COVID-19). Finally, several vaccines with different platforms have been licensed for use in various countries. The injection of these vaccines can lead to a range of unwanted side effects (mild to severe).

Objectives: This study tried to collect the common adverse effects of the most frequently used COVID-19 vaccines in Iran and provide readers with relevant information and statistics.

Methods: This review study collected papers by searching in foreign journals using databases, such as PubMed, PubMed Central, MEDLINE, EBSCO, Google Scholar, and Embase, with the keywords of side effects, COVID-19, vaccine, AstraZeneca, Sputnik V, and Sinopharm, which were published within 2000-2021.

Results: According to available information and studies, vaccines, unlike COVID-19, do not have any threatening and high-risk side effects, and their benefits outweigh their insignificant side effects. A total of 48 full-text studies and 10 valid websites with high citations and up-to-date information were selected. Among the relevant references, 26, 16, and 16 studies performed on AstraZeneca, Sputnik V, and Sinopharm vaccines were chosen for review, respectively.

Conclusions: The findings of this study can reassure readers and be effective in combating misconceptions about vaccination. Undoubtedly, the main solution to overcome the economic damage and social health burden caused by the COVID-19 pandemic is to accelerate global vaccination.
\end{abstract}

Keywords: Side Effects, COVID-19, Vaccine, AstraZeneca, Sputnik V, Sinopharm

\section{Context}

Acute respiratory syndrome coronavirus 2 (SARS-CoV2) appeared in late December 2019 in Wuhan, China, and the disease caused by the virus was named coronavirus disease 2019 (COVID-19). The virus spread rapidly worldwide, creating a deadly pandemic that has continued to this day (1). Iran was one of the first countries which faced the widespread outbreak of COVID-19 and has one of the highest mortality rates regarding the number of cases (2, 3 ). The disease is asymptomatic or mild in most patients. However, a significant percentage of patients develop severe pneumonia, which can lead to hypoxemic respiratory failure, shock, organ dysfunction, and death (4).

The COVID-19 pandemic is arguably the biggest challenge humans have faced since World War II (5). This pandemic poses a serious threat to public health and economic stability around the world, (6) affecting a vast majority of the world's population, and the death rate from SARS-CoV-2 is changing daily (7). During the COVID-19 pandemic, individuals worldwide encounter issues, such as global lockdown, anxiety, stress, and major healthcare challenges (8). Since the outbreak of the disease, various countries have reached the conclusion that the accurate, efficient, comprehensive, reliable, and up-to-date assessment of available information plays a vital role in the effective management of this incident (9). Although several therapeutic compounds and drugs have been recommended to overcome COVID-19, they are often considered supportive treatment options $(10,11)$.

Since January 2020, numerous countries have taken precautionary measures to control the transmission of SARS-CoV-2 in the hope of developing safe and effective vaccines (12). These two main factors might raise concern for some individuals about the possibility of severe side effects after vaccination. However, there have been numerous reports of expected adverse effects (13). Scientists have come to the conclusion that there is no other way to end this pandemic except through public vaccination. Nevertheless, these newly developed vaccines can cause frequent side effects among populations that might decrease qual- 
ity of life and induce some concerns (14).

\section{Objectives}

With this background in mind, the current review aimed to evaluate the short-term side effects associated with COVID-19 vaccines, which are more commonly injected in Iran.

\section{Methods}

The article databases, such as PubMed, Embase, EBSCO, Medine, PubMed Central, and Google Scholar, were used to prepare this review, and 97 English articles and 14 English websites within 2019 - 2021 containing at least one keyword, such as side effects, COVID-19, vaccine, AstraZeneca, Sputnik V, and Sinopharm, were selected and studied. Then, the appropriate studies were reviewed based on the publication date, the name of the first author, and the relevance of the title and its purpose. Finally, 48 English studies and 10 English websites were selected for final analysis and review article writing.

\section{Results}

The SARS-CoV-2 was first observed in Wuhan, China, in December 2019 and endangered the world's health in a very short time (15). According to researchers' statements and the lack of effective antiviral drugs, the development of safe and effective vaccines is the only approach to control the COVID-19 pandemic $(16,17)$. For the development of vaccines against SARS-CoV-2, different platforms have been considered as follows:

(1) Messenger ribonucleic acid-based vaccines, which are the latest generation of produced vaccines: Moderna and Pfizer (Pfizer-BionTech) vaccines fall in this category $(18,19)$.

(2) Vaccines containing viral vectors developed by new methods of biotechnology: Oxford-AstraZeneca, Sputnik V, and Johnson \& Johnson vaccines are of this type $(18,20)$. According to studies, these vaccines have shown a good humoral and cellular immune response after the first dose, and the injection of the second dose develops a stronger and longer-lasting immunity (21-23).

(3) Vaccines containing the killed/inactivated virus that include all or part of a nonliving virus, including Sinopharm, Sinovac, and Bharat Biotech vaccines: $(18,24)$ The inactivated virus vaccine platform has been widely used for over 70 years. In these vaccines, the viruses are neutralized by chemicals, ultraviolet rays, and heat, and finally, a safe vaccine is developed, especially suitable for individuals with a defective immune system. However, they produce lower immune responses than vaccines containing live viruses and require booster doses $(25,26)$. Vaccines containing viral vectors are considered a preventive approach against the pathogen and cause the long-term expression of antigenic proteins. Therefore, they provide a better preventive effect by activating cytotoxic immune $T$ cells (which lead to the removal of virus-infected cells) (27, 28).

(4) Subunit vaccines containing a piece of the virus: These vaccines do not contain genetic structure and are therefore considered safe. Novavax belongs to this group $(19,29)$.

In the following section, the side effects of Sinopharm, AstraZeneca, and Sputnik V vaccines, which have been more frequently used in Iran, will be reviewed:

On August 11, 2020, Russia authorized a vaccine against SARS-CoV-2 called "Sputnik V" manufactured by the Gamaleya Institute in Moscow. The difference between the Sputnik $\mathrm{V}$ vaccine and other vaccines is the use of two different adenovirus vectors instead of a single serotype, namely adenovirus 5 and adenovirus 26. The advantage of this strategy is the production of antibodies against the adenovirus 26 serotype after the first dose and against the adenovirus 5 serotype after the second dose, which improves the immune response (30-32).

The Oxford-AstraZeneca vaccine (ChAdOx1 nCoV-19 vaccine/AZD1222), which uses a nonreplicating chimpanzee adenovirus as a vector to express SARS-CoV-2 spike, was approved by the World Health Organization (WHO) for injection to individuals over 18 and over 65 years of age on February 15, 2021. The vaccine was developed jointly by the University of Oxford, England, and the AstraZeneca Institute $(16,33)$. The European Medicines Agency (EMA) has also fully evaluated and approved the quality, safety, and efficacy of this vaccine (34). The Sinopharm COVID-19 vaccine, developed by the Institute of Biological Products in Beijing, was approved by the WHO on May 7, 2021, as the first Chinese vaccine against COVID-19 $(35,36)$. The information of these three vaccines (ie, AstraZeneca, Sputnik V, and Sinopharm) are available in Table 1.

According to research conducted by the Centers for Disease Control and Prevention, common side effects after vaccination are pain, inflammation, and redness at the injection site, fatigue, fever, chills, muscle pain, headache, and nausea (38). According to experimental and other studies, the injections of each of the two doses of the abovementioned three vaccines (ie, Sputnik V, AstraZeneca, and Sinopharm) have mild to moderate local and systemic side effects, including pain, redness, and inflammation at the injection site, fatigue, headache, chills, muscle pain, joint pain, and fever (39-41). According to a study performed by Kaur et al.,(15) most of the reported reactions after the 


\begin{tabular}{|c|c|c|c|c|c|}
\hline Variables & Type & Efficacy & Dose & Storage & Interval of Vaccine \\
\hline SputnikV & Adenovirus-based & $\begin{array}{l}91.6 \% \text { efficacy against the original strain of the virus; } 100 \% \text { against } \\
\text { severe COVID-19 }\end{array}$ & 2nd & $-18.5^{\circ} \mathrm{C}$ & 21 days apart \\
\hline Sinopharm & Inactivated SARS-CoV-2 (Vero cell) & $\begin{array}{l}\text { 79\% against symptomatic SARS-CoV-2 infection } 14 \text { days or more } \\
\text { after the second dose; } 100 \% \text { against severe COVID-19 }\end{array}$ & 2nd & $2-8^{\circ} \mathrm{C}$ & 21 - 28 days apart \\
\hline AstraZeneca & Adenovirus-based & $\begin{array}{l}76 \% \text { against symptomatic COVID-19; } 100 \% \text { against severe or } \\
\text { critical disease and hospitalization; } 85 \% \text { against symptomatic } \\
\text { COVID-19 in individuals of } 65 \text { years and older }\end{array}$ & 2nd & $2-8^{\circ} \mathrm{C}$ & 28 days apart \\
\hline
\end{tabular}

Abbreviations: SARS-CoV-2, acute respiratory syndrome coronavirus 2; COVID-19, coronavirus disease 2019

injection of Sinopharm and Sputnik V vaccines were mild to moderate, and a small percentage was related to severe complications that all were resolved within 3 - 4 days.

Common reported side effects of the Sputnik V vaccine, according to a study performed by Babamahmoodi et al.,(42) included injection site pain, fatigue, body aches, headache, fever, joint pain, chills, and drowsiness. Diarrhea, depression, skin rash, vomiting, and constipation are also less common symptoms. Rare side effects, such as increased heart rate, itching all over the body, shortness of breath, dryness and bad taste in the mouth, temporary hair loss, runny nose, and sore throat, were reported in less than $0.06 \%$ of study participants. These complications were significantly more frequently observed in women and younger individuals.

The onset of side effects in the subjects was more common within $12-24,24-48,12$, and more than 48 hours to 7 days after vaccination, respectively. However, the duration of experiencing the side effects of the vaccine in most of the participants was less than 3 days (42). According to the findings of Montalti et al.'s study (43), the severity of local and systemic side effects after the second dose of the Sputnik V vaccine was higher than the first dose. Pagotto et al. (44) acknowledged that the side effects of the Sputnik $\mathrm{V}$ vaccine are more common in women and younger individuals, often occur 24 hours after injection and resolve in less than 3 days. According to another study, the side effects of the Sinopharm vaccine resolved within an average of 20 hours after injection (45). Moreover, the prevalence of Sinopharm side effects is in the form of pain at the injection site, fatigue, headache, and discomfort at the injection site, respectively. These side effects are more frequently pronounced in women (46). Other findings regarding the side effects of the Sinopharm vaccine are as follows:

(1) There was a clear linear relationship between vaccination complications and participants' age. These complications are more common in individuals under the age of 49 years than in others (46).

(2) The side effects of the second dose of the vaccine were slightly higher than the first dose $(47,48)$.
(3) Despite the complication of fatigue, there was no significant difference in the possibility of discomfort and redness at the injection site, fever, and headache between men and women (46).

(4) No side effects were observed in $24 \%$ of the subjects after the first dose and 14\% after the second dose (46).

(5) There was no difference between individuals with a history of COVID-19 and individuals with no history of the disease in the onset of symptoms after vaccination (45).

(6) No serious side effects or the need for hospitalization were observed after vaccination (26).

(7) All side effects after the injection of the Sinopharm vaccine were common, predictable, nonlife-threatening, and nonserious (46).

A study conducted by Menni et al. (4) showed that the adverse effects of the AstraZeneca vaccine are common and as follows:

Local symptoms: Feeling discomfort and pain at the injection site, warmth at the injection site, inflammation, redness, and itching

Systemic symptoms: Headache, fatigue, chills, joint pain, and fever (4).

In addition, uncommon symptoms, such as excessive sweating, swollen lymph nodes, pain, loss of appetite, and confusion, have been observed (49). According to the Indian Ministry of Health, the side effects of the AstraZeneca vaccine can last for up to 24 hours and, according to the Food and Drug Administration of India, from a few days to a week $(50,51)$. Other side effects of the AstraZeneca vaccine are as follows:

(1) Compared to men, the severity of complications was higher in women (52).

(2) The severity of complications in individuals under 50 years of age was reported to be higher than others (52).

(3) Individuals with a history of COVID-19 experienced relatively more complications after the vaccine (52).

(4) Individuals with chronic diseases and individuals undergoing medical treatment had slightly fewer local side effects and significantly fewer systemic side effects (52). 
Thrombosis with thrombocytopenia syndrome is another rare complication of the AstraZeneca vaccine (53). Contrary to previous claims, no significant association was observed between the likelihood of this rare complication with age and gender, according to the latest update from the EMA. In this study, which was performed after injecting about 592 million doses of the AstraZeneca vaccine, 1503 cases (about $0.0002 \%$ ) of this complication were reported, $43 \%$ of whom were reported in men and $37 \%$ in individuals over 60 years of age (54). According to the results of all studies, Figures 1-4 are separately presented to compare the prevalence of side effects of the first and second doses of AstraZeneca, Sputnik V, and Sinopharm vaccines.

\subsection{Conclusions}

This study investigated the short-term adverse effects associated with the most commonly used vaccines in Iran, namely AstraZeneca, Sputnik V, and Sinopharm. It was understood that pain at the injection site, fatigue, body pain, and weakness are the most common side effects after injecting the first and second doses of each of the aforementioned vaccines. It seems that the side effects observed following the injection of the AstraZeneca vaccine were more sensible than the other two vaccines. However, none of the reported side effects was life-threatening or worrying, and the benefits of vaccination are still very significant. Vaccination is the only preventive approach against COVID-19 and its consequences. The side effects of vaccination are signs that the vaccine is working well in the body.

\section{Footnotes}

Authors' Contribution: All the authors had a similar role in all stages of conducting this study.

Conflict of Interests: The authors declare that there is no conflict of interest.

Funding/Support: There was no funding or support for this study.

\section{References}

1. Hatmal MM, Alshaer W, Al-Hatamleh MAI, Hatmal M, Smadi O, Taha MO, et al. Comprehensive Structural and Molecular Comparison of Spike Proteins of SARS-CoV-2, SARS-CoV and MERS-CoV, and Their Interactions with ACE2. Cells. 2020;9(12). doi: 10.3390/cells9122638. [PubMed: 33302501]. [PubMed Central: PMC7763676].

2. Raoofi A, Takian A, Akbari Sari A, Olyaeemanesh A, Haghighi H, Aarabi M. COVID-19 Pandemic and Comparative Health Policy Learning in Iran. Arch Iran Med. 2020;23(4):220-34. doi: 10.34172/aim.2020.02. [PubMed: 32271594].

3. Daneshpazhooh M, Mahmoudi H. COVID-19: The experience from Iran. Clin Dermatol. 2021;39(1):23-32. doi: 10.1016/j.clindermatol.2020.12.009. [PubMed: 33972048]. [PubMed Central: PMC7836797].
4. Menni C, Klaser K, May A, Polidori L, Capdevila J, Louca P, et al. Vaccine side-effects and SARS-CoV-2 infection after vaccination in users of the COVID Symptom Study app in the UK: a prospective observational study. Lancet Infect Dis. 2021;21(7):939-49. doi: 10.1016/s14733099(21)00224-3.

5. United Nations Development Programme. Coronavirus. United Nations Development Programme; 2021, [cited 22/9/2021]. Available from: https://www.undp.org/coronavirus.

6. Meo SA, Alhowikan AM, Al-Khlaiwi T, Meo IM, Halepoto DM, Iqbal M, et al. Novel coronavirus 2019-nCoV: prevalence, biological and clinical characteristics comparison with SARS-CoV and MERS-CoV.Eur Rev Med Pharmacol Sci. 2020;24(4):2012-9.

7. Meo SA, Al-Khlaiwi T, Usmani AM, Meo AS, Klonoff DC, Hoang TD. Biological and epidemiological trends in the prevalence and mortality due to outbreaks of novel coronavirus COVID-19. J King Saud Univ Sci. 2020;32(4):2495-9. doi: 10.1016/j.jksus.2020.04.004. [PubMed: 32292261]. [PubMed Central: PMC7144605].

8. Meo SA, Abukhalaf DAA, Alomar AA, Sattar K, Klonoff DC. COVID-19 Pandemic: Impact of Quarantine on Medical Students' Mental Wellbeing and Learning Behaviors. Pakistan J Med Sci. 2020;36(COVID19-S4). doi: 10.12669/pjms.36.COVID19-S4.2809.

9. Azzopardi-Muscat N, Kluge HHP, Asma S, Novillo-Ortiz D. A call to strengthen data in response to COVID-19 and beyond. J Am Med Inform Assoc. 2021;28(3):638-9. doi: 10.1093/jamia/ocaa308. [PubMed: 33275146]. [PubMed Central: PMC7798979].

10. Al-Hatamleh MAI, Hatmal MM, Alshaer W, Rahman E, Mohd-Zahid MH, Alhaj-Qasem DM, et al. COVID-19 infection and nanomedicine applications for development of vaccines and therapeutics: An overview and future perspectives based on polymersomes. EurJ Pharmacol. 2021;896:173930. doi: 10.1016/j.ejphar.2021.173930. [PubMed: 33545157]. [PubMed Central: PMC7857087].

11. Al-Hatamleh MAI, Hatmal MM, Sattar K, Ahmad S, Mustafa MZ, Bittencourt MC, et al. Antiviral and Immunomodulatory Effects of Phytochemicals from Honey against COVID-19: Potential Mechanisms of Action and Future Directions. Molecules. 2020;25(21). doi: 10.3390/molecules25215017. [PubMed: 33138197]. [PubMed Central: PMC7672575].

12. Algaissi AA, Alharbi NK, Hassanain M, Hashem AM. Preparedness and response to COVID-19 in Saudi Arabia: Building on MERS experience. J Infect Public Health. 2020;13(6):834-8. doi: 10.1016/j.jiph.2020.04.016. [PubMed: 32451260]. [PubMed Central: PMC7211706].

13. Public Health Authority. Interim guidelines for the use of SARS-CoV-2 vaccine. Public Health Authority; 2021, [cited 22/9/2021]. Available from: https://covid19.cdc.gov.sa/professionals-health-workers/interimguidelines-for-the-use-of-sars-cov-2-vaccine.

14. Jeskowiak I, Wiatrak B, Grosman-Dziewiszek P, Szelag A. The Incidence and Severity of Post-Vaccination Reactions after Vaccination against COVID-19. Vaccines (Basel). 2021;9(5). doi: 10.3390/vaccines9050502. [PubMed: 34067955]. [PubMed Central: PMC8152224].

15. Kaur RJ, Dutta S, Bhardwaj P, Charan J, Dhingra S, Mitra P, et al. Adverse Events Reported From COVID-19 Vaccine Trials: A Systematic Review. Indian J Clin Biochem. 2021;36(4):427-39. doi:10.1007/s12291-021-00968z. [PubMed: 33814753]. [PubMed Central: PMC7997788].

16. Belete TM. Review on Up-to-Date Status of Candidate Vaccines for COVID-19 Disease. Infect Drug Resist. 2021;14:151-61. doi: 10.2147/IDR.S288877. [PubMed: 33500636]. [PubMed Central: PMC7826065].

17. Samrat SK, Tharappel AM, Li Z, Li H. Prospect of SARS-CoV-2 spike protein: Potential role in vaccine and therapeutic development. Virus Res. 2020;288:198141. doi: 10.1016/j.virusres.2020.198141. [PubMed: 32846196]. [PubMed Central: PMC7443330].

18. Pormohammad A, Zarei M, Ghorbani S, Mohammadi M, Razizadeh $\mathrm{MH}$, Turner DL, et al. Efficacy and Safety of COVID-19 Vaccines: A Systematic Review and Meta-Analysis of Randomized Clinical Trials. Vaccines (Basel). 2021;9(5). doi: 10.3390/vaccines9050467. [PubMed: 34066475]. [PubMed Central: PMC8148145]. 
19. Pardi N, Hogan MJ, Porter FW, Weissman D. mRNA vaccines a new era in vaccinology. Nat Rev Drug Discov. 2018;17(4):261-79. doi: 10.1038/nrd.2017.243. [PubMed: 29326426]. [PubMed Central: PMC5906799].

20. van Riel D, de Wit E. Next-generation vaccine platforms for COVID-19. Nat Mater. 2020;19(8):810-2. doi:10.1038/s41563-020-0746-0. [PubMed: 32704139].

21. Dolzhikova IV, Zubkova OV, Tukhvatulin AI, Dzharullaeva AS, Tukhvatulina NM, Shcheblyakov DV, et al. Safety and immunogenicity of GamEvac-Combi, a heterologous VSV- and Ad5-vectored Ebola vaccine: An open phase I/II trial in healthy adults in Russia. Hum Vaccin Immunother. 2017;13(3):613-20. doi: 10.1080/21645515.2016.1238535. [PubMed: 28152326]. [PubMed Central: PMC5360131].

22. Logunov DY, Dolzhikova IV, Shcheblyakov DV, Tukhvatulin AI, Zubkova OV, Dzharullaeva AS, et al. Safety and efficacy of an rAd26 and rAd5 vector-based heterologous prime-boost COVID-19 vaccine: an interim analysis of a randomised controlled phase 3 trial in Russia. Lancet. 2021;397(10275):671-81. doi:10.1016/s0140-6736(21)00234-8.

23. Tatsis N, Ertl HC. Adenoviruses as vaccine vectors. Mol Ther. 2004;10(4):616-29. doi: 10.1016/j.ymthe.2004.07.013. [PubMed: 15451446]. [PubMed Central: PMC7106330].

24. Sanders B, Koldijk M, Schuitemaker H. Inactivated Viral Vaccines. Vaccine Analysis: Strategies, Principles, and Control. Springer;2015. p. 45-80.

25. Jeyanathan M, Afkhami S, Smaill F, Miller MS, Lichty BD, Xing Z. Immunological considerations for COVID-19 vaccine strategies. Nat Rev Immunol. 2020;20(10):615-32. doi: 10.1038/s41577-020-00434-6. [PubMed: 32887954]. [PubMed Central: PMC7472682].

26. Xia S, Duan K, Zhang Y, Zhao D, Zhang H, Xie Z, et al. Effect of an Inactivated Vaccine Against SARS-CoV-2 on Safety and Immunogenicity Outcomes: Interim Analysis of 2 Randomized Clinical Trials. JAMA. 2020;324(10):951-60. doi: 10.1001/jama.2020.15543. [PubMed: 32789505]. [PubMed Central: PMC7426884].

27. Shirley JL, de Jong YP, Terhorst C, Herzog RW. Immune Responses to Viral Gene Therapy Vectors. Mol Ther. 2020;28(3):709-22. doi 10.1016/j.ymthe.2020.01.001. [PubMed: 31968213]. [PubMed Central: PMC7054714].

28. Graham SP, McLean RK, Spencer AJ, Belij-Rammerstorfer S, Wright $\mathrm{D}$, Ulaszewska $\mathrm{M}$, et al. Evaluation of the immunogenicity of primeboost vaccination with the replication-deficient viral vectored COVID19 vaccine candidate ChAdOx1 nCoV-19. NPJ Vaccines. 2020;5(1):69. doi: 10.1038/s41541-020-00221-3. [PubMed: 32793398]. [PubMed Central: PMC7385486]

29. Kyriakidis NC, Lopez-Cortes A, Gonzalez EV, Grimaldos AB, Prado EO. SARS-CoV-2 vaccines strategies: a comprehensive review of phase 3 candidates. NPJ Vaccines. 2021;6(1):28. doi: 10.1038/s41541-021-00292-w. [PubMed: 33619260]. [PubMed Central: PMC7900244].

30. Petersen E, Wejse C, Zumla A. Advancing COVID-19 vaccines - avoiding different regulatory standards for different vaccines and need for open and transparent data sharing. Int J Infect Dis. 2020;98:501-2. doi: 10.1016/j.ijid.2020.08.043. [PubMed: 32822866]. [PubMed Central: PMC7435296].

31. Balakrishnan VS. The arrival of Sputnik V. Lancet Infect Dis. 2020;20(10). doi: 10.1016/s1473-3099(20)30709-x.

32. Burki TK. The Russian vaccine for COVID-19. Lancet Respir Med. 2020;8(11):85-6. doi: 10.1016/s2213-2600(20)30402-1.

33. News Directory. Breaking stories \& updates. News Directory; 2021, [cited 22/9/2021]. Available from: https://dir.md/content/ astraz/media-centre/press-releases/2021/astrazeneca-covid-19vaccine-authorised-for-emergency-use-by-the-world-healthorganization.html?host=www.astrazeneca.com

34. World Health Organization. The Oxford/AstraZeneca COVID-19 vaccine: what you need to know. World Health Organization; [cited 22/9/2021]. Available from: https://www.who.int/newsroom/feature-stories/detail/the-oxford-astrazeneca-covid-19vaccine-what-you-need-to-know.

35. Wang H, Zhang Y, Huang B, Deng W, Quan Y, Wang W, et al. De- velopment of an Inactivated Vaccine Candidate, BBIBP-CorV, with Potent Protection against SARS-CoV-2. Cell. 2020;182(3):713-721 e9. doi: 10.1016/j.cell.2020.06.008. [PubMed: 32778225]. [PubMed Central: PMC7275151].

36. World Health Organization. WHO lists additional COVID-19 vaccine for emergency use and issues interim policy recommendations. World Health Organization; 2021, [cited 22/9/2021]. Available from: https://www.who.int/news/item/07-05-2021-who-lists-additionalcovid-19-vaccine-for-emergency-use-and-issues-interim-policyrecommendations.

37. Ghiasi N, Valizadeh R, Arabsorkhi M, Hoseyni TS, Esfandiari K, Sadighpour T, et al. Efficacy and side effects of Sputnik V, Sinopharm and AstraZeneca vaccines to stop COVID-19; a review and discussion. Immunopathologia Persa. 2021;7(2):31. doi: 10.34172/ipp.2021.31.

38. Centers for Disease Control and Prevention. What to Expect after Getting a COVID-19 Vaccine. Centers for Disease Control and Prevention; 2021, [cited 22/9/2021]. Available from: https://www.cdc.gov/ coronavirus/2019-ncov/vaccines/expect/after.html.

39. Voysey M, Clemens S, Madhi S, Weckx L, Folegatti P, Aley P. Safety and efficacy of the ChAdOx1 nCoV-19 vaccine (AZD1222) against SARSCoV-2: an interim analysis of four randomised controlled trials in Brazil, South Africa, and the UK. Lancet. 2021;397(10269):99-111. doi: 10.1016/s0140-6736(20)32661-1.

40. Nogrady B. Mounting evidence suggests Sputnik COVID vaccine is safe and effective. Nature. 2021;595(7867):339-40. doi: 10.1038/d41586021-01813-2. [PubMed: 34230663].

41. Xia S, Zhang Y, Wang Y, Wang H, Yang Y, Gao GF, et al. Safety and immunogenicity of an inactivated SARS-CoV-2 vaccine, BBIBP-CorV: a randomised, double-blind, placebo-controlled, phase 1/2 trial. Lancet Infect Dis. 2021;21(1):39-51. doi: 10.1016/s1473-3099(20)30831-8.

42. Babamahmoodi F, Saeedi M, Alizadeh-Navaei R, HedayatizadehOmran A, Mousavi SA, Ovaise G, et al. Side Effects and Immunogenicity Following Administration of The Sputnik V COVID-19 Vaccine Among Health Care Workers; An Observational Study in Iran. Res Square. 2021. doi: 10.21203/rs.3.rs-709499/v1.

43. Montalti M, Solda G, Di Valerio Z, Salussolia A, Lenzi J, Forcellini $M$, et al. ROCCA observational study: Early results on safety of Sputnik V vaccine (Gam-COVID-Vac) in the Republic of San Marino using active surveillance. E Clin Med. 2021;38:101027. doi: 10.1016/j.eclinm.2021.101027. [PubMed: 34505029]. [PubMed Central: PMC8413252].

44. Pagotto V, Ferloni A, Soriano MM, Díaz M, Golde NB, González MI, et al. Active monitoring of early safety of Sputnik V vaccine in Buenos Aires, Argentina. Medicina (Buenos Aires). 2021;81(3):404-14.

45. Jayadevan R, Shenoy R, Ts A. A Survey of symptoms following COVID-19 vaccination in India. medRxiv. 2021. doi: 10.1101/2021.02.08.21251366.

46. Saeed BQ, Al-Shahrabi R, Alhaj SS, Alkokhardi ZM, Adrees AO. Side effects and perceptions following Sinopharm COVID-19 vaccination. Int J Infect Dis. 2021;111:219-26. doi: 10.1016/j.ijid.2021.08.013. [PubMed: 34384899]. [PubMed Central: PMC8351310].

47. Hatmal MM, Al-Hatamleh MAI, Olaimat AN, Hatmal M, Alhaj-Qasem DM, Olaimat TM, et al. Side Effects and Perceptions Following COVID19 Vaccination in Jordan: A Randomized, Cross-Sectional Study Implementing Machine Learning for Predicting Severity of Side Effects. Vaccines (Basel). 2021;9(6). doi: 10.3390/vaccines9060556. [PubMed: 34073382]. [PubMed Central: PMC8229440].

48. El-Shitany NA, Harakeh S, Badr-Eldin SM, Bagher AM, Eid B, Almukadi $\mathrm{H}$, et al. Minor to Moderate Side Effects of Pfizer-BioNTech COVID19 Vaccine Among Saudi Residents: A Retrospective Cross-Sectional Study. Int J Gen Med. 2021;14:1389-401. doi: 10.2147/IJGM.S310497. [PubMed: 33907443]. [PubMed Central: PMC8068468].

49. Open Access Government. What are the side effects of the AstraZeneca vaccine? Open Access Government; 2021, [cited 21/9/2021]. Available from: https://www.openaccessgovernment.org/side-effects-of-theastrazeneca-vaccine/103646/. 
50. Zee Business. Coronavirus Vaccine Covishield: Side effects, Benefits of Serum Institute-AstraZeneca vaccination. Zee Business; 2021, [cited 22/9/2021]. Available from: https://www.zeebiz.com/india/newscoronavirus-vaccine-covishield-side-effects-benefits-of-seruminstitute-astrazeneca-vaccination-145241.

51. Precision Vaccinations. Vaxzevria COVID-19 Vaccine (AstraZeneca). Precision Vaccinations; 2021, [cited 22/9/2021]. Available from: https://www.precisionvaccinations.com/vaccines/vaxzevria-covid19-vaccine-astrazeneca.

52. Riad A, Pokorna A, Mekhemar M, Conrad J, Klugarova J, Koscik M, et al. Safety of ChAdOx1 nCoV-19 Vaccine: Independent Evidence from Two EU States. Vaccines (Basel). 2021;9(6):673. doi: 10.3390/vac- cines9060673. [PubMed: 34207369]. [PubMed Central: PMC8233751].

53. European Medicines Agency. Vaxzevria/COVID-19 Vaccine AstraZeneca: Risk of thrombosis in combination with thrombocytopenia - Updated information. European Medicines Agency; 2021, [cited 21/9/2021]. Available from: https://www.ema.europa.eu/en/medicines/ dhpc/vaxzevriacovid-19-vaccine-astrazeneca-risk-thrombosiscombination-thrombocytopenia-updated.

54. European Medicines Agency. COVID-19 vaccine safety update. European Medicines Agency; 2021, [cited 21/9/2021]. Available from: https: //www.ema.europa.eu/en/documents/covid-19-vaccine-safetyupdate/covid-19-vaccine-safety-update-vaxzevria-previouslycovid-19-vaccine-astrazeneca-8-september-2021_en.pdf. 

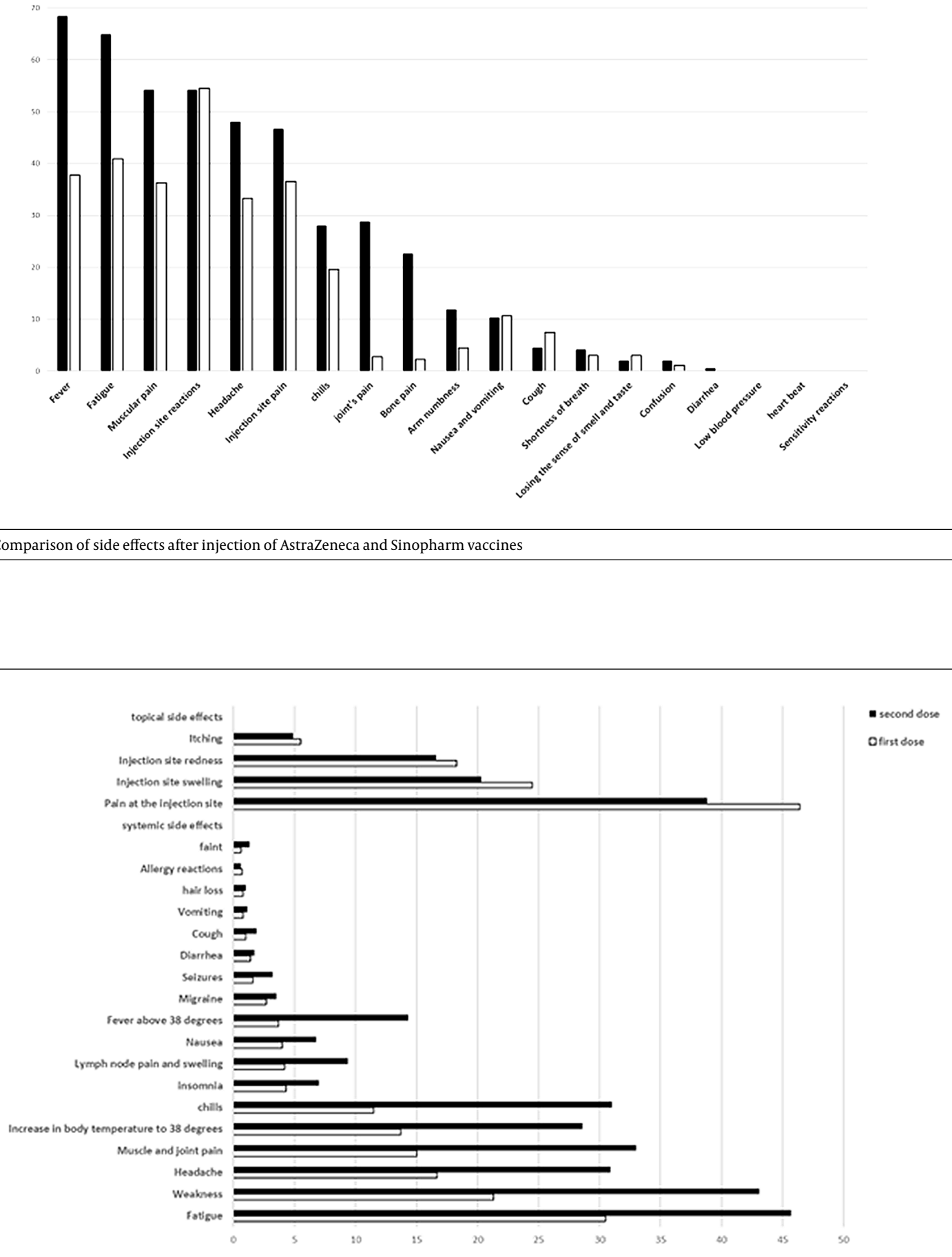

Figure 2. Side effects of AstraZeneca vaccine after the first and second doses 
Uncorrected Proof

Siahvoshi S et al.

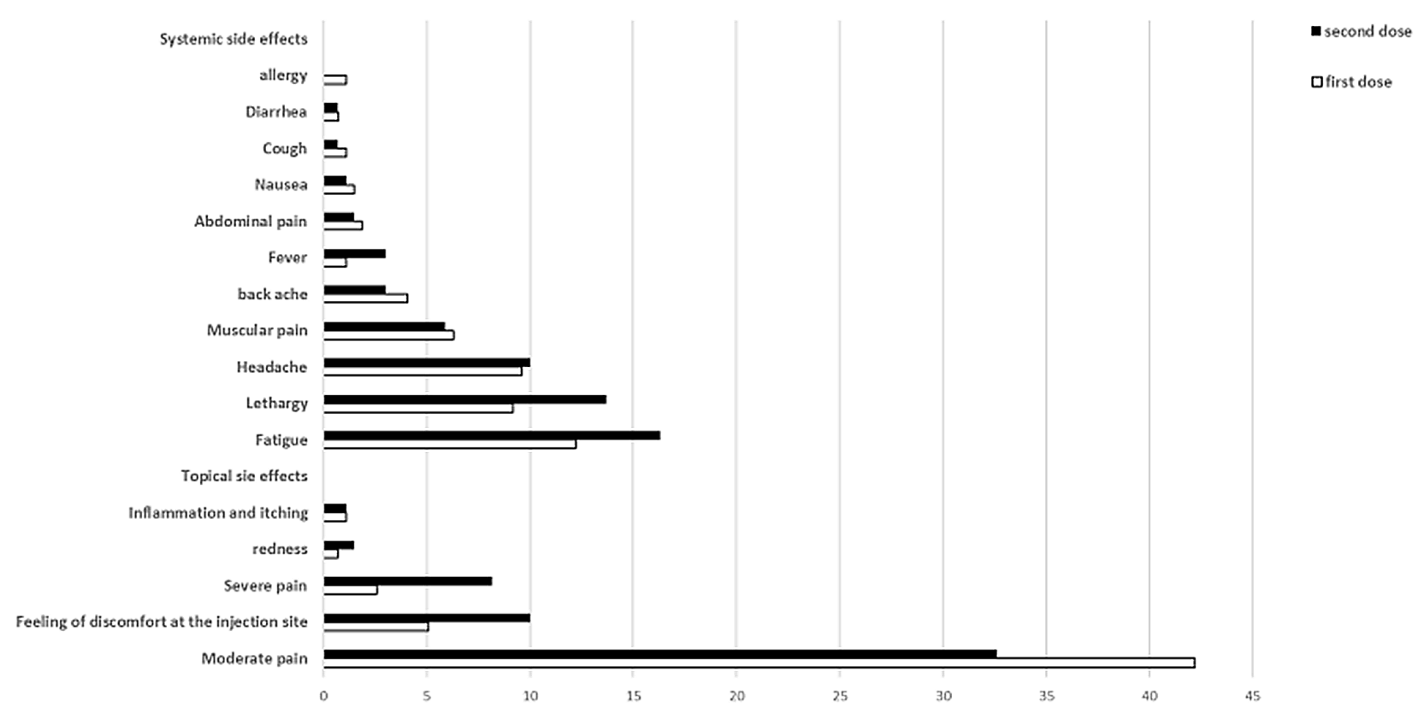

Figure 3. Side effects of Sinopharm vaccine after the first and second doses

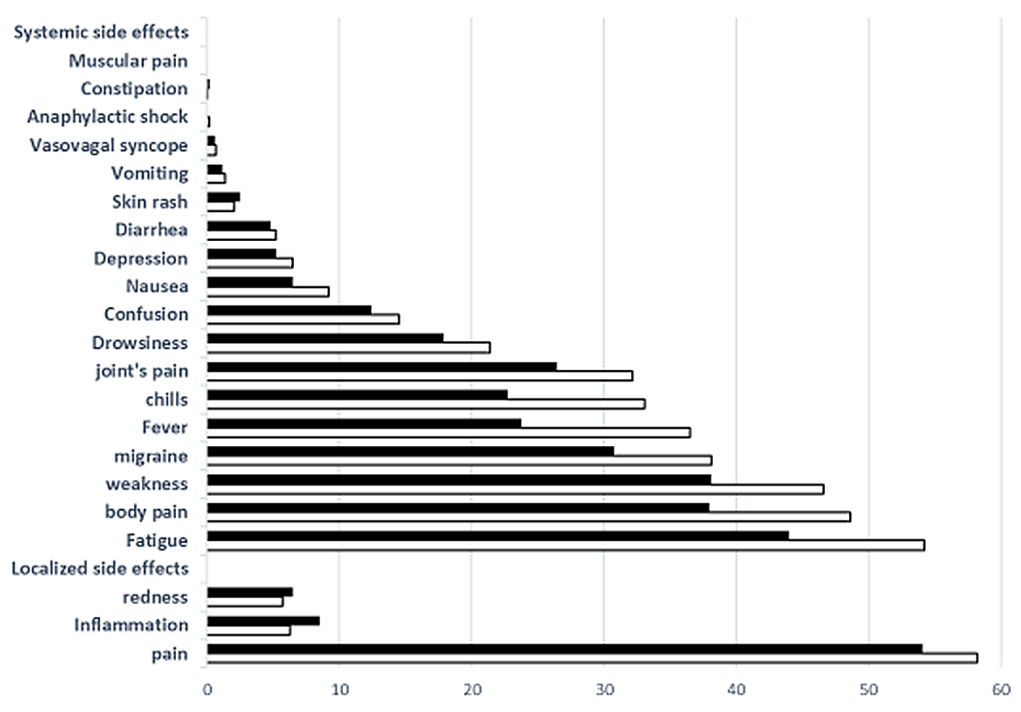

- Second dose ( $N=1042)$

Q First dose $(N=2194)$

Figure 4. Side effects of Sputnik V vaccine after the first and second doses

8

Ann Mil Health Sci Res. 2021;19(4):e120362. 